tised or not. He gives gr. $1 / 6-s s$, and in severe cases gr. $j$, in divided doses, in twenty four hours. If there be much dysphagia, he uses tablet triturates, gr. $\frac{1}{4}$ each, mixed with sugar, and placed dry upon the tongue. With extensive diphtheritic membranes in the fauces he also uses tincture of iron and chlorate of potassium. In threatened heart-failure he uses brandy, caffeine, etc. If the temperature be very high, antipyretics are used, by the mouth or rectum. $\mathrm{He}$ also uses the steam atomizer to produce a moist vapor.

Dr. J. O'Dwyer read a paper on Feeding after Intubation. As a rule, solids and semi-solids are taken much better than liquids after intubation. In a numbe: of cases he had removed the tube in order to give nourishment; but the results were scarcely better than when it was in position. Sumetimes swallowing is easier after than before intubation, on account of the relief afforded to the urgent dyspnoea. The longer the tube remains in position, the easier it generally is to swallow. When the patient is old enough to umderstand, he should be instructed to drink rapidly, and to take as much fluid as possible at one time. Sometimes he uses rectal alimentation, but very seldom uses the stomach-tube. He does not think that food is a cause of pneumonia after intubation, as he has never seen any evidence of milk or other nourishment being found in the minute bronchi. He had known an adult patient to wear a tube for more than ten months, who not only did not have pneumonia, but meanwhile was cured of bron. chitis, which he had when the tube was put in. The secretions, he thinks, are the source of the pneumonia met with after intubation, they being aspirated into the finer bronchi. It is due primarily to the presence of the tube, because it impairs the patient's power of expulsion. As to removing membranes with the forceps, the subglottic division of the tube is of such narrow calibre that it is practically impossible to use this instrument, and he has devised a flexible apparatus for this purpose, somewhat on the principle of the umbrella probang.

Dr. Dillon Brown read a paper on the Statistical Records of Intubation. So far as he had been able to ascertain, 760 cases, by 54 different operators, had been reported; he had tabulated 502 cases-2 II, or 27.07 per cent., had recovered. The average age in fatal cases was 3 years and 2 months; in recoveries, 4 years and $I$ month. In the recoveries intubation was resorted to at an average period of 2 days and 9 hours after symptoms of laryngeal stenosis first ap peared; and in fatal cases at an average period of $I$ day and 19 hours. In the fatal cases the average time between intubation and death was 2 days and 8 hours; in the recoveries the tube was worn on an average for 5 days and $3^{\mathrm{T}} / 2$ hours. O'Dwyer had operated 78 times, Waxham ro6, Dillon Brown 84 , and Huber 47 times. Extension of the membranes to the bronchi was the most frequent cause of death. He believed that pneumonia, when present, was sec ondary in character.

Dr. W. P. Northrup read a paper on the Patholog ical Anatomy of the Respiratory Tract after Death from Laryngeal Diphtheria and Intubation. Since he read his paper on this subject before the Academy in December, in which he reported 87 cases, 20 additional fatal cases had occurred at the New York Foundling Asylum, to which he is pathologist. The cause of this large number during so short a period as four months was the occurrence of diphtheria in connection with a fatal epidemic of measles and scarlet fever. In his former paprer he stated that he had never met with ulcers of the trachea; but in the 20 cases now reported for the first time there were deep ulcers, laying bare the rings of the trachea, in five cases. He had not met with ulceration of any consequence caused by the head of the tube; nothing more than necrosis of the epithelium. He had seen no evidence of aspiration pneumonia, as it had been called, due to the entrance of fluids into the finer bronchi.

Dr. Caillé had performed tracheotomy in 21 cases, with 5 recoveries, and intubation in 16 cases, with 6 recoveries. In his cases of intubation diphtheritic membranes were observed on the nares or pharynx in nine cases. In most of the cases intubation was done rather late. He believed that all the patients would have died without surgical interference, and that one who died would have been saved if intubation had been done earlier. The cause of death after intubation was catarrhal pneumonia in a majority of his cases. Most of the children who died had taken liquids, and of late he had allowed only solids cut up fine, with ice to allay thirst. Since doing this his results were better.

P. B. P.

\section{BERGEON'S METHOD.}

Dear Sir:-Permit me briefly to call attention to a few points in the preparation of gas for Bergeon's treatment. After trying several methods I have, by travelling in a circle, again reached and for the present settled upon, marble chips and $\mathrm{H}_{2} \mathrm{SO}_{4}$ for generating the $\mathrm{CO}_{2}$.

The objection which Dr. Babcock ${ }^{1}$ makes regarding the irritating properties of the gas made from mineral acids is, in my practice, entirely avoided. A gas which made his "nostrils sting" was clearly unfit for use whether there were enteric ulcerations or not. I have discarded $\mathrm{HCl}$. and use only $\mathrm{H}_{2} \mathrm{SO}_{4}$ (C.P.) because the by-products, (such as free chlorine gas) are much harder to get rid of with the former. I use a large mouthed half.gallon generating bottle, in which the marble chips (not dust) are placed. This is connected with the first wash bottle, (of which there are four, half-gallon size,) filled with a strong solution of sodium bicarbonate. Any acid fumes which are carried through would combine with the sodium liberating pure $\mathrm{CO}_{2}$. The next two wash bottles are filled with simple water, unless it is desired to eliminate the $\mathrm{H}_{y} \mathrm{~S}$, which is sometimes formed, in which case Ferric sulphate can be dissolved in one of them. As $\mathrm{H}_{2} \mathrm{~S}$ is not objectionable this is unnecessary. The last wash bottle is filled with the sulphur water, natural or artificial, which it is decided to use. I an using both, and have but little choice, except that the artificial water can be

\footnotetext{
I JourNal, Miay 21, 1887, l). $5^{82}$.
} 
made to impregnate the gas much more strongly with $\mathrm{H}_{2} \mathrm{~S}$, and is much cheaper.

Everything being in readiness, and the gas bag connected. the $\mathrm{H}_{2} \mathrm{SO}$, is poured into the generating hottle. The marble chips should be previously tilted to one side of the bottle and made to remain there as much as possible by gently returning the bottle to the upright position. This prevents too rapid evolu tion of gas; and when the marble in the bottom of the bottle has been utilized fresh marble can be precipitated into the acid by gently shaking it. If the evolution of gas is slow it will be sufficiently pure for all practical purposes. If it bubbles through rapidly and violently the wash bottles will do but little good, and the gas will be pungent and irritating. The number of wash bottles could be increased to half a dozen if it was not thought to be properly washed.

I have had no "colicky pains" reported as a result of the gaseous injections, although I send away from my office bags large enough to last patients from two to three days. I think the reason is that my gas bags are of heavy rubber, through which osmosis must be difficult, and to any appreciable extent, perhaps impossible.

I have nothing to report clinically, except that all the patients upon whom I have used this treatment have shown improvement in some of the symptoms. I am not prepared to say that there has been arrest in any case, though one patient has used it faithfully for seven weeks." It is, of course, too soon to ex press a final verdict; and we can only work on, hoping that it will prove something more than an. other chimera added to the long list of "consump. tion specifics." If its power of controlling symptoms should prove to be reliable, and prove to be its only value, Bergeon would still cleserve well of his genereration. Respectfully,

Fort Wayne, Ind., May 24, 1837 . G. W. MCCaskey, M.D.

\section{TAPPING VS. INCISION OF THE CYST IN} OVARIOTOMY.

Dear Sir:-In the letter of Dr. N. Senn, published in The Journal of June 4 , there occur on page $64 \mathrm{I}$, in reference to an ovariotomy by $\mathrm{Mr}$. Langton, the following remarks: "The abdomen was opened by an incision through the linea alba about four inches in length, and the cyst tapped with the large trocar of Spencer Wells. 'The puncture was followed by a free escape of fluid along the sides of the trocar, and some of it entered the abdominal cavity, and the wound was freely irrigated with the cyst contents. i... It has always seemed to me that the use of a large trocar with a truncated cutting edge in tapping a tense cyst is attended invariably by extravasation of thid, and consequently increases the risk of peri. tonitis. When the cyst contents are fluid, the patient should either be placed upon her side during the tapping, and proper precautions adopted to prevent entrance of fluid into the abdominal cavity, or the cyst should be emptied sufficiently to bring it

\footnotetext{
2 This patient died June sth.
}

into the wound by the use of a small trocar or by aspiration. In case the contents are colloid they will escape through no tube, and incision of the cyst with the patient on her side, and traction upon the cyst wall, so as to keep it in uninterrupted accurate contact with the abdominal wall, are the only measures which will accomplish the desired object with safety."

I heartily endorse the foregoing suggestions of Prof. Senn. Having for many years observed the inadequacy of the various forms of trocar in preventing the escape of cystic fluid along the sides of the instrument, I have latterly dispensed with it entirely in some cases, and opened the cyst with a knife instead. Dr. Carl Braun uses the same method.

The cyst having been exposed and the investigation of adhesions finished, a dry piece of antiseptic gauze folded into a pad of eight or more thicknesses is placed between the cyst and the incised abdominal wall on the side towards the operator. The patient is then rolled strongly over on the corresponding side, and the cyst pressed forward into the wound by the hand of an assistant applied against the flank. At a selected spot a small incision or puncture is made with the point of knife, the opening being subsequently enlarged to any extent desired. Besides the dry pad, and the intra abdominal pressure of the cyst, an additional guard against the entrance of cystic fluid into the peritoneal cavity may be furnished in the follow. ing manner: Take a piece of tin plate eight or ten inches long and four inches wide, and bend it length. wise into the form of a half cylinder. The end of this trough, held against the cyst immediately below the point of the intended incision, will serve to convey any fluid which may possibly escape from the cut away from the field of operation. 'This device will be found of great service in whatever manner the cyst may be opened.

So soon as the cyst wall becomes sufficiently relaxed, I seize it in folds with strong dull-toothed vulsella, and by constant traction keep the opening and collapsing cyst outside the abdominal wound until complete extraction is accomplished.

27r Michigan Ave., Chicago.

\section{A. Reeves Jackson, M.D.}

\section{BOOK REVIEWS.}

Diseases of Women. A handbook for physicians and Students. By DR. F. Winckei, Professor of Gynacology, and Director of the Royal University Clinic in Munich. Authorized Translation by J. H. Wirmiamson, M.D., Resident Physician Allegheny General Hospital, Allegheny, Pennsylvania. Under the supervision of Theophilus Parvin, M.D., Professor of Obstetrics and Diseases of Women and Children in Jefferson Medical College, Philadelphia. 8vo., pp. xxix-674. Philadelphia: P. Blakiston, Son \& Co. I887.

It is a little more than a year since Professor Winckel's work was issued in Leipsig. As a teacher of obstetrics and gynecology he has but few rivals, 